\title{
Nucleon spin structure at Jefferson Lab
}

Angela Biselli

Fairfield University, abiselli@fairfield.edu

Follow this and additional works at: https://digitalcommons.fairfield.edu/physics-facultypubs Copyright 2011 AIP Publishing

The final publisher PDF has been archived here with permission from the copyright holder. This article may be downloaded for personal use only. Any other use requires prior permission of the author and AIP Publishing. The following article appeared in Biselli, A., \& CLAS collaboration. (2011, October). Nucleon spin structure at Jefferson Lab. In AIP Conference Proceedings Vol. 1374, No. 1, pp. 227-230 and may be found at https://doi.org/10.1063/ 1.3647131.

\section{Repository Citation}

Biselli, Angela, "Nucleon spin structure at Jefferson Lab" (2011). Physics Faculty Publications. 130. https://digitalcommons.fairfield.edu/physics-facultypubs/130

\section{Published Citation}

Biselli, A., \& CLAS collaboration. (2011, October). Nucleon spin structure at Jefferson Lab. AIP Conference Proceedings Vol. 1374, No. 1, pp. 227-230.

This item has been accepted for inclusion in DigitalCommons@Fairfield by an authorized administrator of DigitalCommons@Fairfield. It is brought to you by DigitalCommons@Fairfield with permission from the rightsholder(s) and is protected by copyright and/or related rights. You are free to use this item in any way that is permitted by the copyright and related rights legislation that applies to your use. For other uses, you need to obtain permission from the rights-holder(s) directly, unless additional rights are indicated by a Creative Commons license in the record and/or on the work itself. For more information, please contact digitalcommons@fairfield.edu. 


\section{Nucleon spin structure at Jefferson Lab}

Angela Biselli, and The CLAS collaboration

Citation: AIP Conference Proceedings 1374, 227 (2011);

View online: https://doi.org/10.1063/1.3647131

View Table of Contents: http://aip.scitation.org/toc/apc/1374/1

Published by the American Institute of Physics 


\title{
Nucleon spin structure at Jefferson Lab
}

\author{
Angela Biselli \\ for the CLAS collaboration
}

Fairfield University, Fairfield, CT 06828

\begin{abstract}
In the past decade an extensive experimental program to measure the spin structure of the nucleon has been carried out in the three halls at Jefferson Lab. Using a longitudinally polarized beam scattering off longitudinally or transversely polarized ${ }^{3} \mathrm{He}, \mathrm{NH}_{3}$ and $\mathrm{ND}_{3}$ targets, the double spin asymmetries $A_{\|}$and $A_{\perp}$ were measured, providing data of impressively high precision that gives a better understanding of the structure of the nucleon in the deep inelastic scattering and the valence quarks regions. The virtual photon asymmetries $A_{1,2}$ and polarized structure functions $g_{1,2}$ were also extracted for the proton, neutron and deuteron over large kinematic ranges, allowing the extraction of first moments and the testing of sum rules and duality.
\end{abstract}

Keywords: Spin structure, Nucleon Spin

PACS: $13.60 . \mathrm{Hb}, 13.88 .+\mathrm{e}, 14.20 . \mathrm{Dh}$

\section{INTRODUCTION}

After the "spin crisis" in 1980, large experimental programs have been devoted to unraveling the spin structure of the nucleon. Inclusive inelastic scattering of polarized electrons off polarized nucleons is a powerful tool to study the polarized structure functions and their moments. For a longitudinally polarized beam the measured asymmetries are

$$
A_{\|}=\frac{\sigma^{\uparrow \Uparrow}-\sigma^{\downarrow \Uparrow}}{\sigma^{\uparrow \Uparrow}+\sigma^{\downarrow \Uparrow}} \text { and } A_{\perp}=\frac{\sigma^{\uparrow \Rightarrow}-\sigma^{\downarrow \Rightarrow}}{\sigma^{\uparrow \Rightarrow}+\sigma^{\downarrow \Rightarrow}}
$$

where $\sigma^{\uparrow \Uparrow}\left(\sigma^{\uparrow \Downarrow}\right)$ is the cross sections for beam and target polarization oriented parallel (antiparallel), and $\sigma^{\uparrow \Rightarrow}\left(\sigma^{\downarrow \Rightarrow}\right)$ is the cross section for a transversely polarized target with the electron spin aligned antiparallel (parallel) to the beam direction. The asymmetries $A_{\|}$and $A_{\perp}$ are related to the virtual photon asymmetries $A_{1}$ and $A_{2}$ by

$$
\frac{A_{\|}}{D}=A_{1}+\eta A_{2} \text { and } \frac{A_{\perp}}{d}=A_{1}+\zeta A_{2}
$$

where $\eta=\varepsilon Q /\left(E-E^{\prime} \varepsilon\right), \zeta=\eta(1+\varepsilon) /(2 \varepsilon), D=\left(1-E^{\prime} \varepsilon / E\right) /(1+\varepsilon R), d=D \sqrt{2 \varepsilon /(1+\varepsilon)}, R=\sigma_{L} / \sigma_{T}$ is the ratio of longitudinal and transverse virtual photon-absorption cross sections, $Q^{2}$ is the squared 4-momentum transfer, and $\varepsilon=\left(1+2 \frac{\vec{q}^{2}}{Q^{2}} \tan ^{2} \frac{\theta_{e}}{2}\right)^{-1}$ is the degree of transverse photon polarization. The asymmetry $A_{1}$ is related to the difference of virtual photo-absorption cross sections for total helicity between photon and nucleon of $1 / 2$ and $3 / 2$, while $A_{2}$ is related to the interference term between the transverse and longitudinal photon-nucleon amplitudes.

Given the virtual photon asymmetries, one can extract the spin structure function $g_{1}$ and $g_{2}$ using:

$$
g_{1}\left(x, Q^{2}\right)=\frac{\tau}{1+\tau}\left(A_{1}+\frac{1}{\sqrt{\tau}} A_{2}\right) F_{1}\left(x, Q^{2}\right) \text { and } g_{2}\left(x, Q^{2}\right)=\frac{\tau}{1+\tau}\left(-A_{1}+\sqrt{\tau} A_{2}\right) F_{1}\left(x, Q^{2}\right)
$$

where $\tau=v^{2} / Q^{2}, v$ is the energy transfer of the virtual photon, and $F_{1}$ is the unpolarized structure function. The spin structure function $g_{2}$ contains information about higher twists.

A quantity of particular interest is the first moment of $g_{1}$

$$
\Gamma_{1}\left(Q^{2}\right)=\int_{0}^{1} g_{1}\left(x, Q^{2}\right) d x
$$

At high $Q^{2}, \Gamma_{1}^{p-n}$ is related to the spin carried by the quarks as stated by the Bjorken sum rule. For $Q^{2} \rightarrow 0$ this quantity becomes $\frac{Q^{2}}{2 M^{2}} I_{G D H}$, where $I_{G D H}$ is the Gerasimov-Drell-Hearn sum rule and is equal to $-\kappa^{2} / 4$, where $\kappa$ is the nucleon anomalous magnetic moment.

12th International Conference on Meson-Nucleon Physics and the Structure of the Nucleon (MENU 2010)
AIP Conf. Proc. 1374, 227-230 (2011); doi: 10.1063/1.3647131

(C) 2011 American Institute of Physics 978-0-7354-0934-7/\$30.00 


\section{SELECTED RESULTS}

The virtual photon asymmetry $A_{1}$ was measured in all three experimental Halls at Jefferson Lab in different kinematic ranges and for different nucleons. The Hall A experiment [1,2] used the High Resolution Spectrometer (HRS) with a momentum resolution of $2 \times 10^{-4}$ and a ${ }^{3} \mathrm{He}$ target, in which more than $87 \%$ of the spin is carried by the neutron, thereby providing an effective polarized neutron target. The target was oriented both longitudinally and transversely with respect to the direction of the beam, allowing the measurement of both $A_{\|}$and $A_{\perp}$. The result for $A_{1}^{n}$ shows the first evidence of $A_{1}^{n}$ becoming positive and large at high x, consistent with the relativistic constituent quark model (RCQM) predictions, the LSS 2001 perturbative quantum chromo-dynamics (PQCD) fit to previous data and a global NLO QCD analysis of deep inelastic scattering (DIS) data [1]. The Hall B experiment, known as the EG1 experiment [3, 4], used the CLAS detector, which gives a nearly $2 \pi$ acceptance, achieving a kinematic coverage from 0.05 up to $5 \mathrm{GeV}^{2}$ in $Q^{2}$ and from 1.08 to $3 \mathrm{GeV}$ in $W$. The targets were longitudinally polarized, solid, 1 -cm-long, ammonia targets, ${ }^{15} \mathrm{NH}_{3}$ and ${ }^{15} \mathrm{ND}_{3}$, polarized up to $70 \%$ and $40 \%$ respectively via dynamic nuclear polarization [5]. Preliminary results are shown in Figure 1 for the virtual photon asymmetry $A_{1}$ for the proton (left) and deuterium (right) averaged in the deep inelastic region $Q^{2}>1 \mathrm{GeV}^{2}$ and $W>2 \mathrm{GeV}$. These preliminary results confirm the trend of $A_{1}$ to exceed the SU(6) limit at $x=1$ already found in [4]. At higher $x$, where the valence quarks are expected to dominate, data are most consistent with the hyperfine perturbed quark model [6], but also very close to other models that consider different scenarios of $\mathrm{SU}(6)$ symmetry breaking [7].
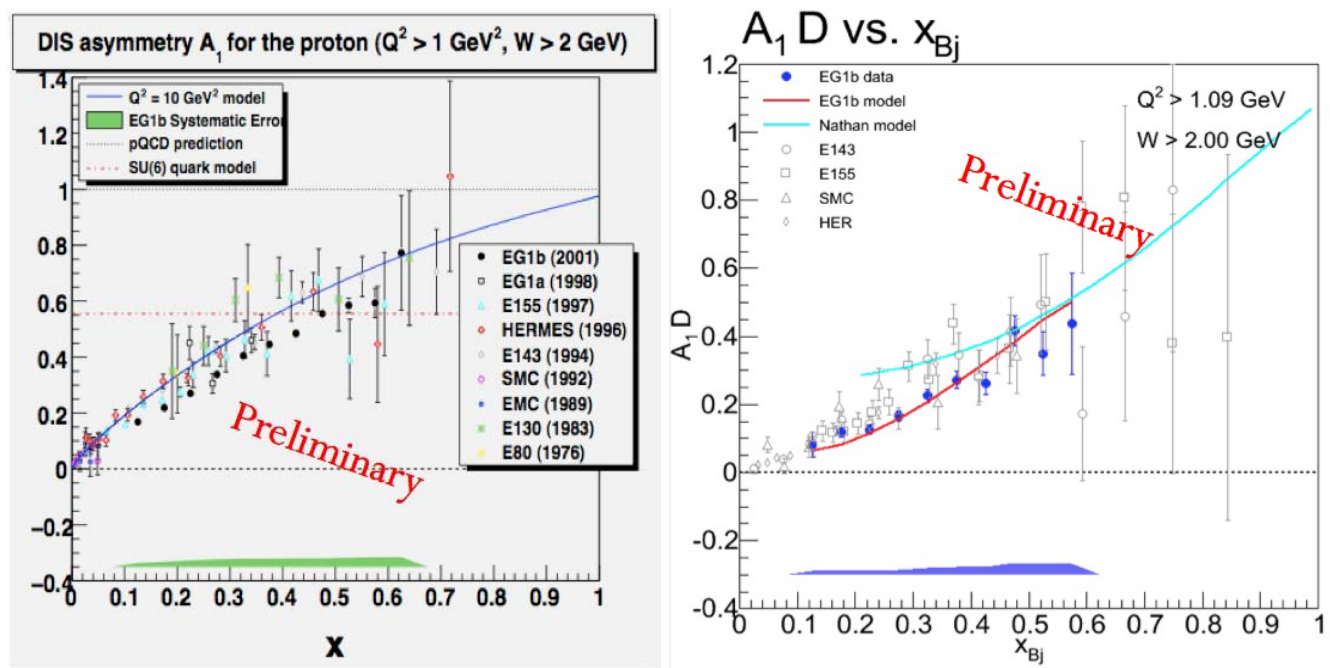

FIGURE 1. Virtual photon asymmetry $A_{1}$ vs. $x$ for proton (left) and deuteron (right).

The structure function $g_{1}$ can be extracted from the virtual photon asymmetries using a parameterization of the world's data for the unpolarized structure function $F_{1}$. Figure 2 shows a sample of the results of the EG1 experiment for the deuterium polarized structure function $g_{1}$ for several $Q^{2}$ bins. Similar results were found for the proton. As can be seen at low $Q^{2}$ the $\Delta(1232)$ resonance drives the structure function towards negative values, due to dominance of the spin-3/2 amplitude. At higher $Q^{2}, g_{1}$ becomes positive everywhere.

The virtual photon asymmetry $A_{2}$ and the polarized structure function $g_{2}$ were measured both in Hall A [2] and Hall C [8] due to the ability of running there with a transversely polarized target. The RSS experiment in Hall C used the High Momentum Spectrometer and longitudinally and transversely polarized ammonia targets ${ }^{15} \mathrm{NH}_{3}$ and ${ }^{15} \mathrm{ND}_{3}$. The experiment focused on the resonance region $1.09<W<1.91 \mathrm{GeV}$ at an average $Q^{2}$ of $1.3 \mathrm{GeV}^{2}$. The structure function $g_{2}$ for the proton is clearly non-zero, providing strong evidence of higher-twist contributions as shown in Figure $3[8]$.

The extensive kinematic range of these measurement allows the extraction of the first moments. Figure 4 shows the first moment of the structure function $g_{1}$ (see Eq. 4) for the proton using EG1 data. The world data parameterization was used to include the unmeasured part to the integral down to $x=0.001$. The low $Q^{2}$ data points are consistent with the $I_{G D H}$ limit, and are in fairly good agreement with the phenomenological model by Burkert and Ioffe [9] that parameterizes the transition between the real photon point and the DIS regime using measured pion electro- and photo-production resonance amplitudes. The comparison of data at low $Q^{2}$ with the ChPT calculation of Ji (dashed 


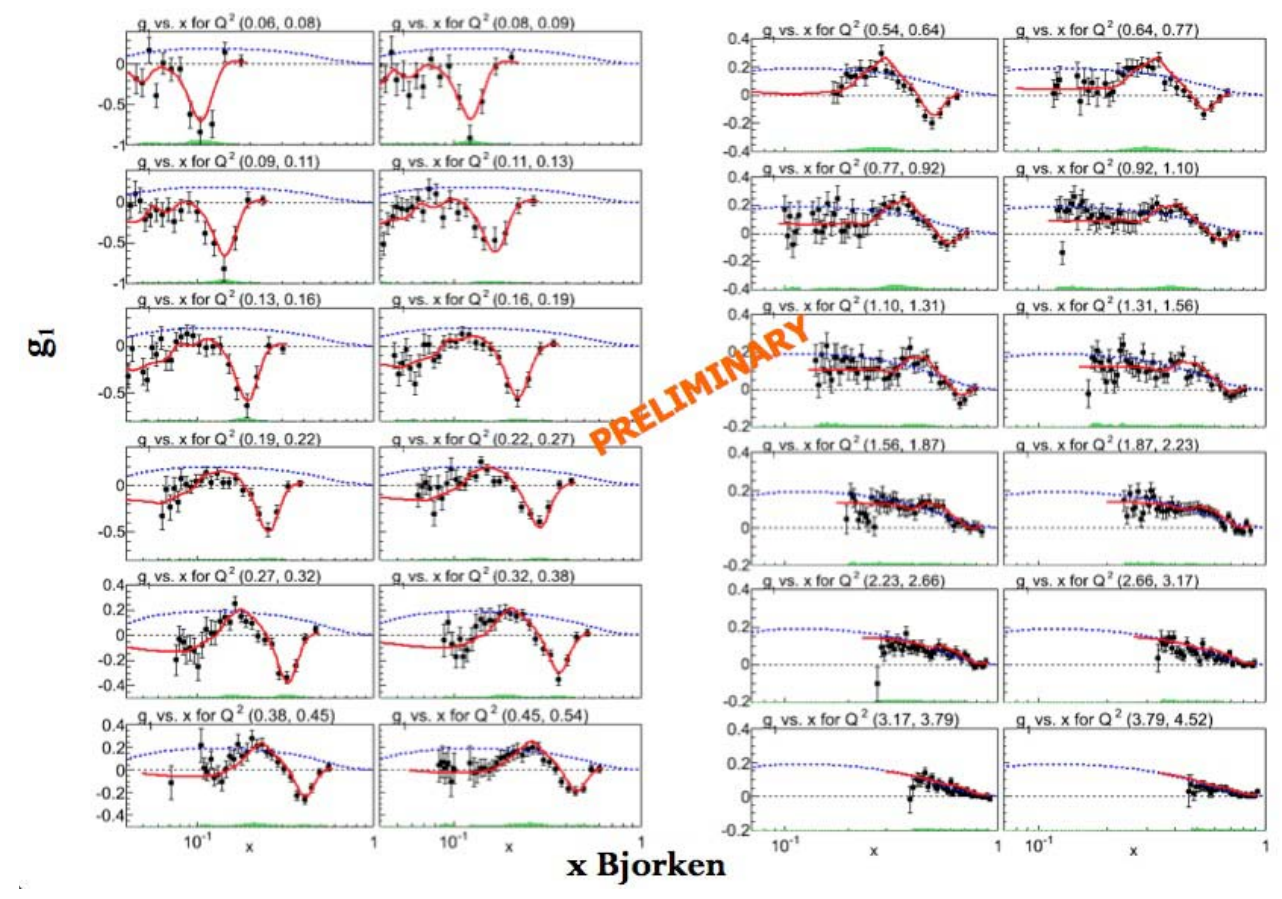

FIGURE 2. The structure function $g_{1}$ vs $x$ for several $Q^{2}$ bins from 0.06 to $2.23 \mathrm{GeV}^{2}$.

curve) [10] shows a disagreement; however, this calculation is an expansion up to $Q^{4}$ and a fit of the data up to $Q^{8}$ shows agreement with Ji's $Q^{4}$ coefficient and a non negligible $Q^{6}$ term even at $Q^{2}<0.1 \mathrm{GeV}^{2}$ [3]. The right panel of Figure 3 shows the first moment of the structure function $g_{1}$ for the neutron using Hall A data [11] including, the latest preliminary results at low $Q^{2}$, which are also in good agreement with the phenomenological model by Burkert and Ioffe.

The experimental program on the spin structure at Jefferson Lab has produced unprecedentedly precise measurements of the spin structure functions $A_{1,2}, g_{1,2}, \Gamma_{1}$ and related quantities for the proton, neutron and deuteron. New results are expected from more recent experiments such as the Hall B EG4 experiment and the Hall C SANE ex-
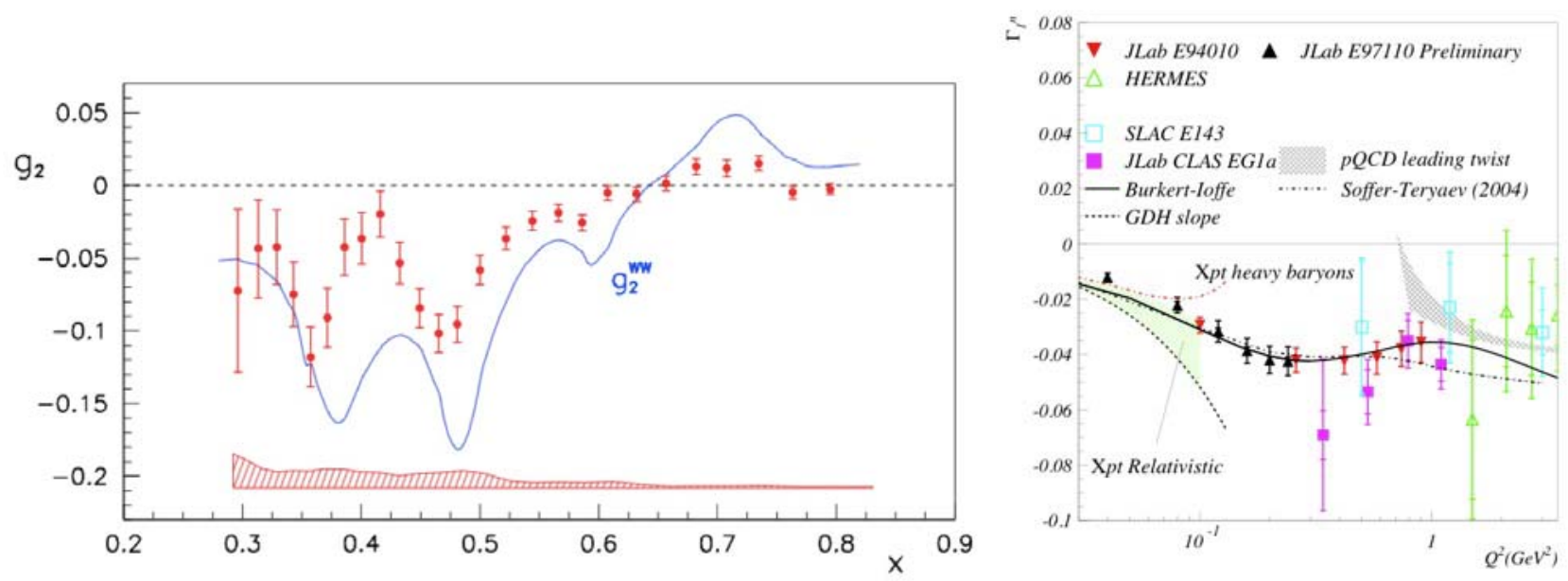

FIGURE 3. The structure function $g_{2}$ vs. $x$ for proton (left) [8] and first moment of the structure function $g_{1}$ for the neutron using Hall A data [11] (right). 

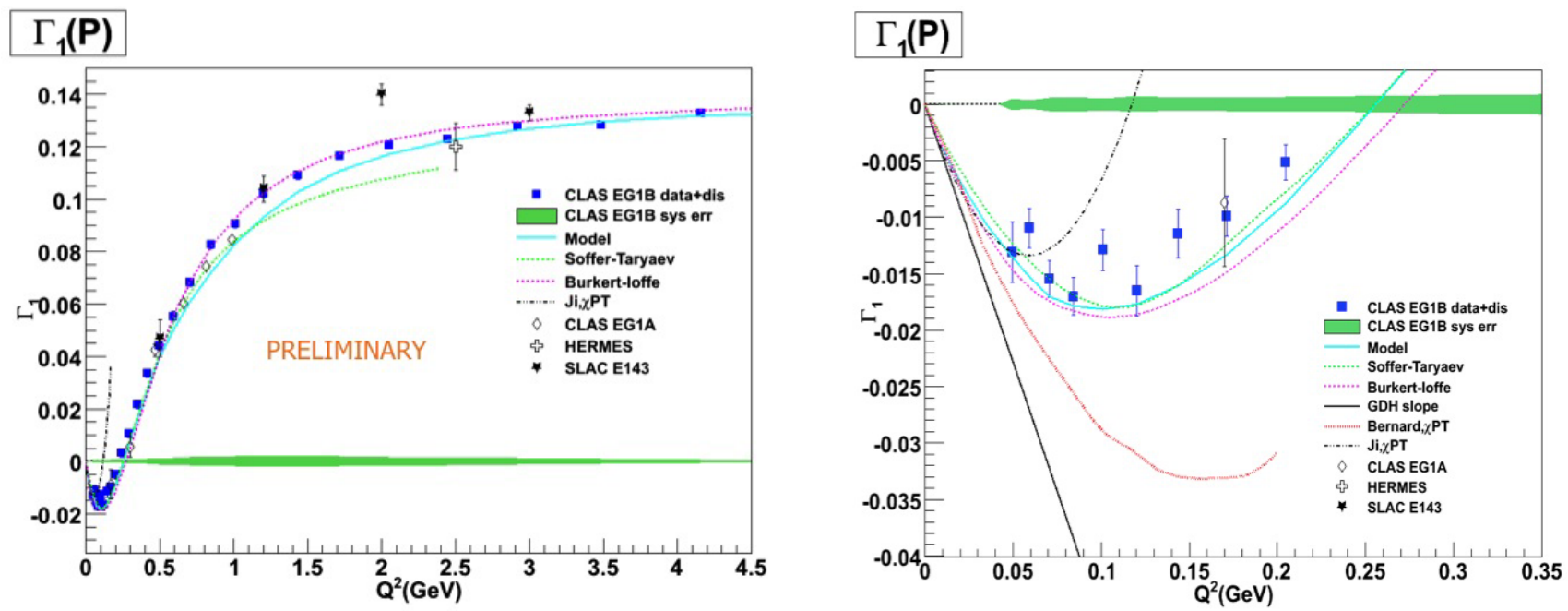

FIGURE 4. First moment of $g_{1}$ for the proton $\left(\Gamma_{1}^{p}\right)$ vs $Q^{2}$. The left plot shows the full range in $Q^{2}$ while the right plot shows the lower $Q^{2}$ region. The figure shows the new preliminary data from EG1 (squares) and the previous EG1 (diamonds), HERMES (crosses) and SLAC E143 (stars) results. Phenomenological predictions (Burkert-Ioffe, Soffer-Teryaev, ChPT) and the GDH slope are also shown.

periment. The EG4 experiment, as a result of an additional Cherenkov counter placed at low angles, will extend the measurement of $\Gamma_{1}$ down to $0.015 \mathrm{GeV}^{2}$, allowing a better test of the ChPT calculations. The SANE experiment which uses the novel large solid angle electron telescope BETA detector will extract $A_{1}$ at high x, study the structure function $g_{1}$ and $g_{2}$ and twist 3 effects from their moments. In addition new experiments are planned for the $12 \mathrm{GeV}$ upgrade of the accelerator. The higher energy and the improved detectors will increase the statistical precision of the current measurements, will extend the measurements to kinematic regions at the moment not accessible, such as high $x$, and will allow the extraction of fundamental quantities such as parton distribution functions.

\section{ACKNOWLEDGMENTS}

We would like to thank the staff of the Accelerator and Physics Divisions at the Jefferson Lab for their outstanding efforts to provide us with the high quality beam and the facilities for data analysis. This work was supported by the U.S. Department of Energy and the National Science Foundation, the French Commissariat a l'Energie Atomique, the Italian Istituto Nazionale di Fisica Nucleare, and the Korean Science and Engineering Foundation. Jefferson Science Associates (JSA) operates the Thomas Jefferson National Accelerator Facility for the United States Department of Energy under contract DE-AC05- 84ER40150.

\section{REFERENCES}

1. X. Zheng et al. [Jefferson Lab Hall A Collaboration], Phys. Rev. Lett. 92, 012004 (2004).

2. X. Zheng et al. [Jefferson Lab Hall A Collaboration], Phys. Rev. C 70, 065207 (2004) [arXiv:nucl-ex/0405006].

3. Y. Prok et al. [CLAS Collaboration], Phys. Lett. B 672, 12 (2009).

4. K. V. Dharmawardane et al. [CLAS Collaboration], Phys. Lett. B 641, 11 (2006).

5. C. D. Keith et al., Nucl. Instrum. Meth. A 501, 327 (2003).

6. N. Isgur, Phys. Rev. D 59, 034013 (1999).

7. F. E. Close and W. Melnitchouk, Phys. Rev. C 68, 035210 (2003).

8. F. Wesselmann et al. [RSS Collaboration], Phys. Rev. Lett., 98, 132003, (2007).

9. V. D. Burkert and B. L. Ioffe, Phys. Lett. B 296, 223 (1992).

10. X. D. Ji, C. W. Kao and J. Osborne, Phys. Lett. B 472, 1 (2000).

11. M. Amarian et al. [Jefferson Lab E94-010 Collaboration], Phys. Rev. Lett. 92, 022301 (2004). 